\title{
DESIGN OF AN ARC-BASED THRUST-OPTIMIZED NOZZLE CONTOUR
}

\author{
K. Schomberg ${ }^{1}$, J. Olsen ${ }^{1}$, A. Neely ${ }^{2}$, and G. Doig ${ }^{1,3}$ \\ ${ }^{1}$ School of Mechanical and Manufacturing Engineering \\ UNSW Australia \\ Sydney, NSW 2052, Australia \\ ${ }^{2}$ School of Engineering and Information Technology \\ UNSW Canberra \\ Canberra, ACT 2600, Australia \\ ${ }^{3}$ Aerospace Engineering Department \\ California Polytechnic State University \\ San Luis Obispo, CA 93407, USA
}

\begin{abstract}
A total of ten arc-based nozzle contours have been numerically compared to an existing thrust-optimized design. Verification and validation of the numerical model was achieved using published experimental data and the resulting numerical uncertainty of the model relative to thrust was less than $0.10 \%$. Analysis of the arc-based nozzles indicated that the contour angles had the greatest effect on thrust. Optimization of the contour angles suggested that an increase in thrust coefficient of $0.25 \%$ could be achieved compared to the existing thrust-optimized nozzle. The result shows that an arc-based design method may be used to produce an equivalent thrust-optimized nozzle.
\end{abstract}

\section{INTRODUCTION}

The supersonic nozzle in a rocket propulsion system is generally designed to maximize the exhaust velocity and, therefore, the thrust coefficient. A technique to produce a 'thrust-optimized' nozzle for this purpose was generated over half a century ago, by applying calculus of variations to the method of characteristics [1]. The use of this method became particularly popular when it was later simplified by Rao and is often denoted as a Rao nozzle contour [2]. Rao also showed that an optimal nozzle contour can be approximated using a skewed parabola if a small decrease in thrust is accepted [3]. Later work indicated that direct design methods could be used to design a nozzle contour that produces

(C) The Authors, published by EDP Sciences. This is an open access article distributed under the terms of the Creative Commons Attribution License 4.0 (http://creativecommons.org/licenses/by/4.0/). 
a near-optimal thrust coefficient $[4,5]$. The use of direct methods can be advantageous in terms of design control and transferability, where the simplest direct method utilizes circular arcs in the construction of a nozzle contour.

The concept of using circular arcs for the design of a nozzle contour is well known [6], although existing documentation regarding arc-based nozzles is limited within the public domain. The initial use of circular arcs in a supersonic nozzle was shown to produce 'much, but not all' of the possible thrust gain compared to conical designs [6]. It was eventually concluded that arc-based nozzles were inferior to conventional designs by up to $1.0 \%$ of total thrust [7]. However, the arc-based designs used in this particular analytical analysis constrained the exit angle and were $10 \%-35 \%$ shorter than a common rocket nozzle contour. Comparatively, the use of circular arcs for design of the nozzle by manipulating the contour angles in solid rocket motors was found to be approximately $( \pm 0.50 \%)$ equal to optimum thrust nozzles produced by a number of direct methods [8].

In this work, an attempt to address the previous ambiguity in the definition and analysis of circular arc-based nozzle contours has been made. This was achieved by numerically comparing a range of arc-based nozzle contours to an existing thrust- optimized nozzle. The LEA thrust-optimized parabola (LEA TOP) contour was selected for this purpose due to the availability of the nozzle geometry and published experimental data [9]. An equal or greater thrust coefficient in an arc-based design relative to the LEA TOP nozzle would suggest the potential of circular arcs in the design of nozzle contours has been misdiagnosed and that the arc-based design method may be used to generate an equivalent thrust-optimized nozzle.

\section{NUMERICAL MODEL}

The behavior of all nozzles considered in this work was evaluated using the commercial ANSYS Fluent 14.5 finite volume code. An implicit axisymmetric pressure-based solver was selected in conjunction with second-order upwind schemes to generate all results. Although initially developed for low-speed flows, the solver has been extended to compressible flow conditions [10] and has modeled similar flow fields with a high degree of accuracy [11, 12]. A Reynoldsaveraged approach to turbulence was used due to the quasi-steady nature of the experimental flow conditions [9]. The use of air as the working fluid was maintained and adherence to the ideal gas law was assumed in all cases. A stagnation temperature of $288 \mathrm{~K}$ was used in all cases and the low stagnation enthalpy permitted the use of a three-coefficient Sutherland approximation to describe viscosity [13]. Solution convergence was accepted when a negligible difference in mass flux was recorded between the inlet and outlet boundaries and a varia- 


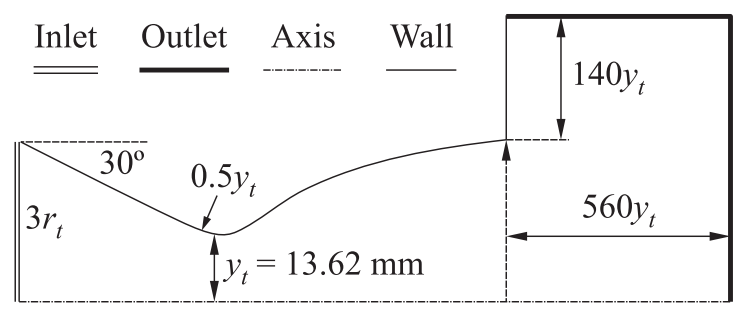

Figure 1 Numerical domain with outlet and inlet dimensions (not to scale)

tion in all flow parameters of less than $0.01 \%$ was achieved over $10^{3}$ iterations. This condition was generally obtained after $10^{4}$ iterations and corresponded to normalized residual levels of $10^{-3}$ for continuity and $10^{-6}$ for momentum and energy.

The existing LEA TOP nozzle geometry was replicated using Rao's method $[3,9]$. The inlet geometry was created by maintaining the radius of the expansion curve through up to $30^{\circ}$ upstream of the throat and a contraction ratio of 9 used to allow the static conditions at the inlet to approach the stagnation values. The exhaust domain was sized with respect to the experimental setup to ensure that the numerical model could be accurately verified and validated, and a schematic of the numerical domain is given in Fig. 1. Verification and validation of the numerical model was achieved by establishing grid independence and considering the turbulence closure model with respect to the published experimental data [9]. The experimental uncertainty was estimated from the combined nonlinearity, hysteresis, and repeatability error of the Kulite XCQ-062-25A pressure sensors used for the experiments [9].

\subsection{Grid Independence}

Discretization of the numerical domain was achieved using a fully-structured scheme comprized of quadrilateral elements. A multiblock strategy was employed in all cases to ensure a nondimensional wall distance $(y+)$ of less than 1 at the nozzle wall. In addition, orthogonal elements were maintained immediately downstream of the nozzle exit for a total distance of four times the spanwise exit dimension $\left(\approx 22 y_{t}\right)$. Grid independence was established by comparing the static pressure distribution and thrust coefficient across three grids of increasing refinement. The grid density was controlled by setting a maximum element aspect ratio in both the streamwise $(x)$ and spanwise $(y)$ directions immediately downstream of the nozzle exit. These limits were varied to produce a coarse $(100 \times 50)$, fine $(50 \times 25)$, and extrafine $(30 \times 20)$ level of grid density containing $4,7.5$, and $19 \cdot 10^{5}$ elements, respectively. The solutions used to determine 
Table 1 Thrust coefficient relative to grid density at low-altitude $\left(C_{F}\right)$, ideal $\left(C_{F 0}\right)$, and vacuum $\left(C_{F \infty}\right)$ conditions

\begin{tabular}{lccc}
\hline \multicolumn{1}{c}{ Grid } & $C_{F}$ & $C_{F 0}$ & $C_{F \infty}$ \\
\hline Coarse & 1.269 & 1.605 & 1.649 \\
Fine & 1.269 & 1.605 & 1.648 \\
Extrafine & 1.269 & 1.605 & 1.648 \\
\hline
\end{tabular}

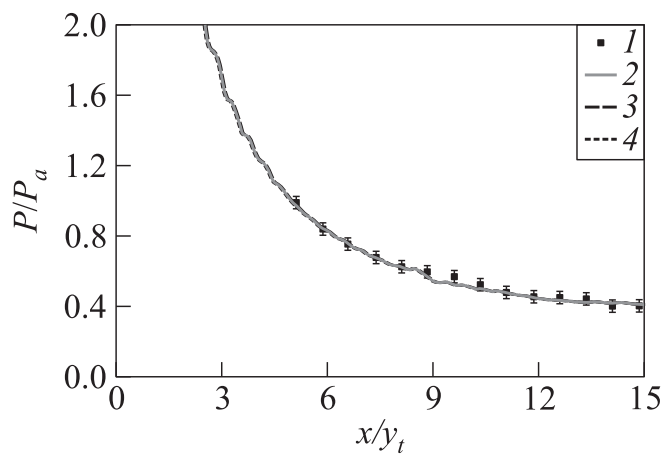

Figure 2 Static pressure distribution relative to grid density at low-altitude conditions: 1 LEA TOP; 2 - coarse; 3 - fine; and $4-$ extrafine vacuum conditions, the thrust coefficient predicted in the coarse grid solution was $0.06 \%$ greater than that of the fine and extrafine grids. The effect of grid density on the static pressure distribution was negligible and the distribution
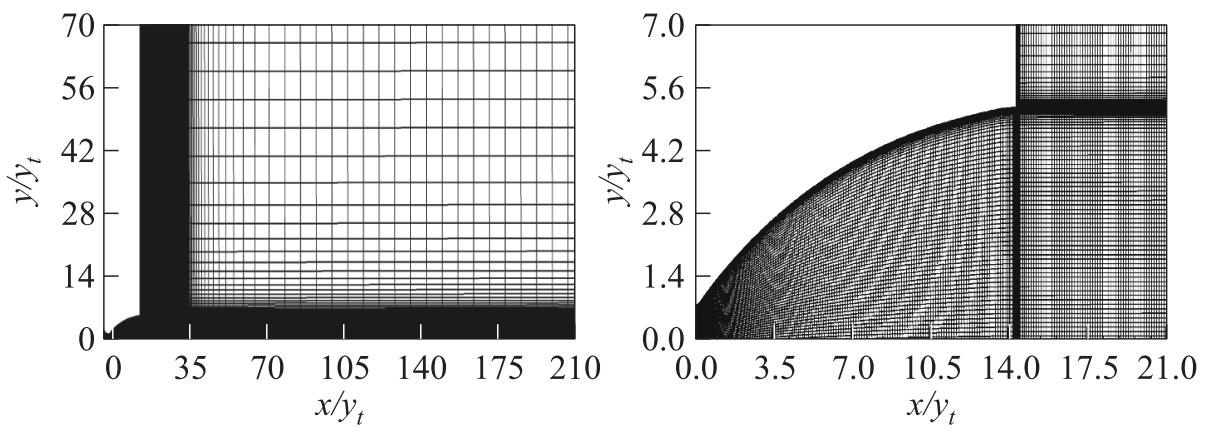

Figure 3 Numerical domain with fine grid 
was within the bounds of experimental uncertainty for all data points. The fine grid, shown in Fig. 3, was selected to ensure an accurate thrust coefficient at all operating conditions and used in all future models.

\subsection{Turbulence Model}

Selection of the turbulence closure model was determined by comparing an inviscid solution to the one-equation Spalart-Allmaras (SA) model and the two-equation shear stress transport (SST) model [16]. The static pressure distribution at low-altitude flow conditions and the thrust coefficient was again used for the verification process and is shown in Fig. 4. In addition, the thrust coefficient was evaluated at low-altitude, ideal, and vacuum conditions and is given in Table 2 .

A close agreement between the experimental and numerical values was observed in the static pressure distribution. This was expected due to the quasi-inviscid nature of the unseparated flow at lowaltitude conditions. The

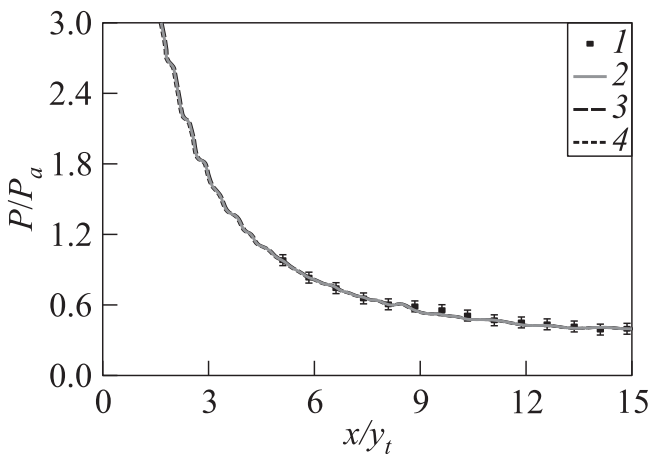

Figure 4 Static pressure distribution relative to turbulence closure model at low-altitude flow conditions: 1 - LEA TOP; 2 - inviscid; 2 - SA; and $4-\mathrm{SST}$

Table 2 Thrust coefficient relative to turbulence model

\begin{tabular}{lccc}
\hline Model & $C_{F}$ & $C_{F 0}$ & $C_{F \infty}$ \\
\hline Inviscid & 1.278 & 1.614 & 1.657 \\
SA & 1.269 & 1.605 & 1.648 \\
SST & 1.268 & 1.606 & 1.649 \\
\hline
\end{tabular}
thrust coefficient was $0.50 \%-$ $0.80 \%$ greater in the inviscid solution relative to the SA and SST solutions, highlighting the influence of the boundary layer on thrust. Comparatively, the variation in thrust coefficient between the SA and SST solution was $0.05 \%-0.08 \%$ across all conditions.

The SA turbulence model was chosen to generate all future results due to a small reduction in computational time compared to the SST model.

The numerical uncertainty of the model with respect to thrust coefficient was $\pm 0.001(0.10 \%)$ for all conditions. This range provided the precision threshold for comparison between the existing LEA TOP and arc-based nozzles. 


\section{ANALYSIS}

\subsection{Arc-Based Design Method}

The design of an arc-based contour can be achieved using a finite series of circular arc segments for both the expansion and turning curves in the nozzle. This design method will only be described in brief here since it has been previously outlined in detail in [11]. In this work, both the expansion and turning curves were produced from a single arc segment. The contour notation used in the generation of all arc-based nozzle contours is given in Fig. 5 .

An expansion curve radius $\left(r_{t m}\right)$ of $0.5 y_{t}$ was used in all designs, consistent with the existing LEA TOP nozzle. The turning curve radius $\left(r_{m n}\right)$ can then be expressed by considering the curve end points as two right-angled triangles, and calculated using the following equation:

$$
r_{m n}=\frac{y_{n}-y_{m}}{\cos \theta_{n}-\cos \theta_{m}}=\frac{x_{n}-x_{m}}{\sin \theta_{m}-\sin \theta_{n}} .
$$

If the expansion curve radius is given, the radius of the turning curve becomes a function of four parameters: $x_{n}, y_{n}, \theta_{m}$, and $\theta_{n}$. To produce a solution, three of these parameters must be defined. This process is suited to the design of supersonic nozzles as the desired exit Mach number (and, therefore, spanwise dimension) or maximum streamwise length is often specified. In addition, the normalization of all dimensional variables is inherent to the process itself and can be used to ensure scalability between designs.

The definition of the contour angles can be achieved through the use of a baseline design. In this case, it is convenient to use a 15 degree constant angle contour

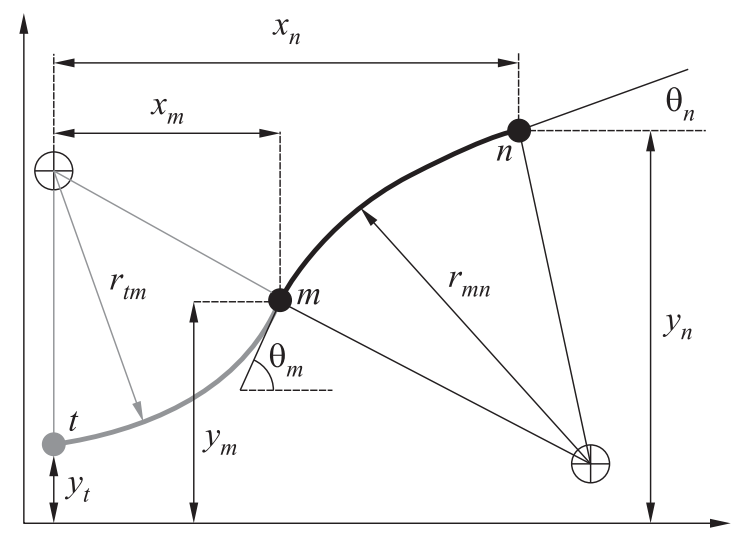

Figure 5 Notation used for the generation of an arc-based nozzle contour 
as it is often the standard used to compare streamwise lengths between nozzle designs [17]. If a constant spanwise dimension at the exit is assumed, a function can be derived that approximates the ratio of streamwise length to contour angles by use of a simple power function. The subscript ' $c$ ' has been used to represent the baseline (constant angle) design and the expression that equates the streamwise length and contour angles is given below:

$$
\frac{x_{n}-x_{m}}{x_{c}}=\frac{\sin \theta_{m}-\sin \theta_{n}}{\cos \theta_{n}-\cos \theta_{m}} \frac{\sin \theta_{c}}{\cos \theta_{c}} \approx \frac{2 \theta_{c}}{\theta_{m}+\theta_{n}} .
$$

Note that this expression is based on the tangent of the angle approaching the angle itself and, therefore, the accuracy is reduced when high angles are used. However, it has previously been shown that the approximation is inherently conservative and the difference for all reasonable nozzle contour angles is less than $5 \%[11]$.

\subsection{Preliminary Analysis}

The geometric parameters of the LEA TOP nozzle were taken directly from the existing data [9]. Two arc-based equivalent nozzles were generated by maintaining either the spanwise or streamwise dimensions with the LEA TOP nozzle and the resulting contours denoted as the ARC_Xn and ARC_Yn variants, respectively. The existing LEA TOP streamwise distance was then substituted in Eq. (1) to give an angle sum of $33.25^{\circ}$. A design angle sum of $34^{\circ}$ was, therefore, selected for use in this work to eliminate the effect of the expansion curve and to ensure a conservative arc-based nozzle length. Two additional arc-based nozzle variants were then produced by using an exit angle and inflection angle that was consistent with the LEA TOP nozzle, resulting in the ARC_$\theta \mathrm{m}$ and ARC_$\theta \mathrm{n}$ variants, respectively.

The geometric parameters of all preliminary nozzle variants are given in Table 3 .

Table 3 Geometric parameters of preliminary nozzle variants

\begin{tabular}{|c|c|c|c|c|}
\hline Model & $y_{n}$ & $x_{n}$ & $\theta_{m}$ & $\overline{\theta_{n}}$ \\
\hline LEA_TOP & $5.51 r_{t}$ & $15.0 r_{t}$ & 34 & 4 \\
\hline ARC_Yn & $6.15 r_{t}$ & $15.0 r_{t}$ & 34 & 4 \\
\hline ARC_Xn & $5.51 r_{t}$ & $13.1 r_{t}$ & 34 & 4 \\
\hline ARC_$\theta \mathrm{m}$ & $5.51 r_{t}$ & $14.8 r_{t}{ }^{*}$ & 30 & 4 \\
\hline ARC_ $\theta \mathrm{n}$ & $5.51 r_{t}$ & $14.8 r_{t}{ }^{*}$ & 34 & 0 \\
\hline
\end{tabular}

${ }^{*}$ Reduced length due to higher angle sum. 
Table 4 Thrust coefficient relative to each nozzle variant

\begin{tabular}{|c|c|c|c|}
\hline Model & $C_{F}$ & $C_{F 0}$ & $C_{F \infty}$ \\
\hline LEA_TOP & 1.269 & 1.605 & 1.648 \\
\hline ARC_Yn & 1.256 & 1.595 & 1.638 \\
\hline ARC_Xn & 1.260 & 1.596 & 1.639 \\
\hline ARC_ $\theta \mathrm{m}$ & 1.270 & 1.606 & 1.649 \\
\hline ARC_ $\theta \mathrm{n}$ & 1.262 & 1.598 & 1.641 \\
\hline
\end{tabular}

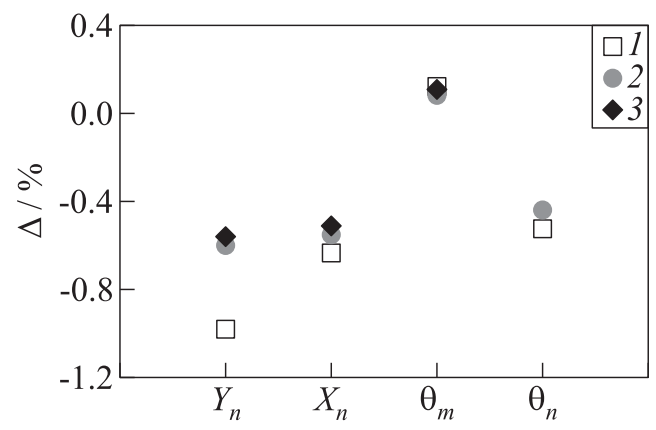

Figure 6 Difference in thrust coefficient relative to the LEA TOP nozzle: $1-C_{F} ; 2-$ $C_{F 0}$, and $3-C_{F \infty}$
All preliminary arc-based nozzle variants were geometrically advantageous compared to the LEA TOP design. An increase in the spanwise dimension of $11 \%$ was achieved for an equivalent streamwise length in the ARC_Yn variant, which would theoretically improve thrust at vacuum conditions. In addition, a reduction in streamwise length of $13 \%$ was achieved in the ARC_Xn design. The conservative angle sum of $34^{\circ}$ used for both angle variants resulted in a reduction in streamwise length of approximately $1.0 \%$. Comparison between the initial arc variants and the existing LEA TOP contour was made using thrust coefficient at low-altitude, ideal, and vacuum flow conditions. Thrust coefficient relative to each nozzle variant and the relative difference compared to the LEA TOP nozzle are given in Table 4 and Fig. 6, respectively.

All contour variants apart from the ARC_ $\theta \mathrm{m}$ nozzle returned a thrust coefficient that was lower than the LEA TOP nozzle. This difference ranged between $0.40 \%-0.60 \%$ at ideal and vacuum operating conditions to $0.50 \%-1.0 \%$ at lowaltitude conditions. The finding was in agreement with previous work [7], which states that a reduction in thrust will occur in any equivalent arc-based nozzle contour. However, it is worth noting that the thrust coefficient values presented here were not scaled relative to contour length in spite of a reduction in length of over $10 \%$ in the ARC_Xn variant. As contour length generally implies nozzle weight, the thrust coefficient in various contours may be compared in the global context of the entire propulsion system by scaling the thrust with respect to an engine thrust-to-weight ratio [11]. In this case, the thrust coefficient of the ARC_Xn nozzle becomes greater than the LEA_TOP nozzle whenever the mass of the nozzle is more than $5 \%$ of the engine [11].

An increase in thrust coefficient of $0.06 \%-0.08 \%$ was observed in the ARC_$\theta \mathrm{m}$ nozzle variant at all operating conditions. The result was particularly significant as a common approach when defining an equivalent arc-based nozzle is to simply 
minimize the exit angle. This particular method of arc-based nozzle design is currently used in the industry standard TDK (two-dimensional kinetics) code [18] and may explain the ambiguity in previous work when assessing the merit of arcbased nozzle contours $[7,8]$. The difference between the LEA TOP and ARC_ $\theta \mathrm{m}$ nozzle contours was within the level of uncertainty in the numerical model and indicated that the arc-based method may be capable of producing an equivalent thrust-optimized contour.

\subsection{Contour Angle Optimization}

The use of an inflection and exit angle sum in the arc-based design method ensures that an equivalent contour will always be produced if this angle sum is maintained. This process naturally facilitates a range of valid inflection and exit angle combinations when applied to any set of design conditions. To determine the potential of the arc-based design method in producing a contour equivalent to the LEA TOP nozzle, the range of valid contour angles was assessed with respect to thrust coefficient at ideal operating conditions.

The design spectrum in this case ranged from the ARC_ $\theta \mathrm{n}$ variant to an equivalent conical nozzle which for the length ratio of the LEA TOP nozzle resulted in a constant divergence angle of $17^{\circ}$. The remaining angle range was split incrementally into four or five total equivalent contours where additional thrust curves derived from three and seven data points were used to determine convergence of the optimum contour geometry. Each equivalent contour was defined using the angle coefficient $\left(C_{\theta}\right)$ defined in the following equation:

Table 5 Variation in thrust coefficient relative to contour angles

\begin{tabular}{lcll}
\hline$\theta_{m}$ & $\theta_{n}$ & $C_{\theta}$ & $C_{F 0}$ \\
\hline 17 & 17 & 1 & 1.590 \\
21.25 & 12.75 & 0.6 & 1.602 \\
22.67 & 11.33 & 0.5 & 1.605 \\
25.5 & 8.5 & 0.33 & 1.609 \\
28.33 & 5.67 & 0.2 & 1.609 \\
29.75 & 4.25 & 0.14 & 1.607 \\
34 & 0 & 0 & 1.598 \\
\hline
\end{tabular}

Thrust coefficient at ideal conditions was used for the optimization process in an attempt to consider the entire range of operating conditions. Table 5 outlines the variation in thrust coefficient relative to contour angles and the thrust curves relative to the LEA TOP nozzle and the variation in optimum angle coefficient are given in Fig. 7 and Table 6 , respectively.

The difference between the equivalent conical and highest performing contour was $1.2 \%$, highlighting the considerable effect of the contour angles on thrust coefficient. Observation of the relative thrust difference indicated that the optimum angle coefficient was between 0.2 and 0.33 . Convergence of the relative thrust 


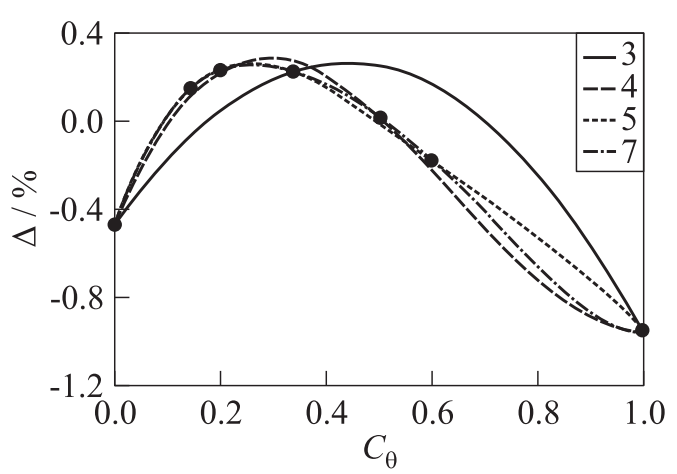

Figure 7 Relative thrust difference curves using a given number of data points with respect to contour angle

Table 6 Variation in optimum angle coefficient and resulting thrust increase

\begin{tabular}{cccrc}
\hline $\begin{array}{c}\text { Number } \\
\text { of points }\end{array}$ & $C_{\theta}$ & $\theta_{m}$ & $\theta_{n}$ & $\Delta, \%$ \\
\hline 3 & 0.436 & 23.7 & 10.3 & 0.26 \\
4 & 0.286 & 26.4 & 7.6 & 0.28 \\
5 & 0.258 & 27.0 & 7.0 & 0.25 \\
7 & 0.261 & 27.0 & 7.0 & 0.25 \\
\hline
\end{tabular}

magnitude and angle coefficient for the design parameters considered here was achieved using five data points. The use of three and four data points skewed the curve towards high angle coefficients, and a greater maximum thrust difference was predicted when four points were used. The result suggests that an increase in thrust of $0.25 \%$ could be achieved in an equivalent arc-based nozzle compared to the existing LEA TOP design.

\section{DISCUSSION AND CONCLUDING REMARKS}

The design of an arc-based thrust-optimized nozzle contour was shown to be possible. The use of an existing thrust-optimized nozzle (LEA TOP) for comparison presented a challenge geometrically, as only three of the four main contour parameters could be maintained in any equivalent arc-based nozzle. Furthermore, the selection of these geometric parameters was shown to have a considerable $(>0.50 \%)$ effect on thrust, where only one out of the four arc-based nozzle variants returned a thrust coefficient that was equivalent to that of the LEA TOP nozzle.

The evidence of a clear relationship between nozzle geometry and thrust coefficient was supported by the existing literature regarding arc-based nozzle analysis on two main counts. Firstly, by suggesting that the ambiguity in previ- 
ous findings may be linked to how the arc-based contour is defined as opposed to an inherent flaw within the concept itself. And secondly, by highlighting that an arc-based nozzle generated using current industry standard design methods is likely to produce a thrust coefficient that is lower than a thrust-optimized nozzle produced by existing techniques.

Representation of the arc-based design method in terms of nozzle contour angles enabled the spanwise and streamwise dimensions to be maintained with respect to a calculated angle sum. Optimization of the contour angles with respect to the thrust coefficient was completed at ideal conditions using a total of seven equivalent arc-based contours. Convergence of an optimal arc-based geometry was shown to occur using five design points and suggested that a thrust increase of $0.25 \%$ could be achieved relative to the existing LEA TOP nozzle. The result challenges the current perception of arc-based nozzle design and indicated that the use of optimal contour angles in an arc-based nozzle may produce equal or greater thrust compared to existing methods.

\section{REFERENCES}

1. Guderley, G., and E. Hantsch. 1955. Beste Formen fur Aschsensymmetrische Uberschallschubdusen. Z. Flugwissensch. 3:305-313.

2. Rao, G. 1958. Exhaust nozzle contour for optimum thrust. Jet Propulsion 28(6):377-382

3. Rao, G. 1960. Approximation of optimum thrust nozzle contour. ARS J. - Am. Rocket Soc. J. 30(6):561.

4. Hoffman, J. 1987. Design of compressed truncated perfect nozzles. J. Propul. Power 3(2):150-156.

5. Dumnov, G., and N. Ponomarev. 2000. Comparison of two methods used for contouring launch vehicle's engine nozzles. AIAA Paper No. 2000-3170.

6. Sutton, G. 2005. History of liquid propellant rocket engines. Library of flight ser. American Institute of Aeronautics and Astronautics. 91-92.

7. Sternin, L. 2000. Analysis of the thrust characteristics of jet nozzles designed by various methods. Fluid Dyn. 35(1):123-131.

8. Davis, D. 1982. Investigation of optimization techniques for solid rocket motor nozzle contour. AIAA Paper No. 82-1188.

9. Nguyen, A., H. Deniau, S. Girard, and T. Alziary De Roquefort. 2003. Unsteadiness of flow separation and end-effects regime in a thrust-optimized contour rocket nozzle. Flow Turbul. Combust. 75:161-181.

10. Koutsavdis, E., and G. Stuckert. 2002. A numerical investigation of the flow characteristics of plug nozzles using fluent. AIAA Paper No. 2002-0511.

11. Schomberg, K., G. Doig, and J. Olsen. 2014. Design of high area nozzle contours using circular arcs. AIAA Paper No. 2014-3999. 
12. Schomberg, K., G. Doig, J. Olsen, and A. Neely. 2014. Geometric analysis of linear expansion-deflection nozzle geometry at highly overexpanded flow conditions. AIAA Paper No. 2014-4001.

13. Sutherland, W. 1893. The viscosity of gases and molecular force. Philos. Mag. 36:507-531.

14. Spalart, P., and S. Allmaras. 1992. A one-equation turbulence model for aerodynamic flows. Rech. Aerospatiale 1(5):5-21.

15. Stark, R., and G. Hagemann. 2007. Current status of numerical flow prediction for separated nozzle flows. 2nd European Conference for Aerospace Sciences. 1-8.

16. Menter, F. 1994. Two equation eddy-viscosity turbulence models for engineering applications. AIAA J. 32(8):1598-1605.

17. Sutton, G., and O. Biblarz. 2001. Rocket propulsion elements. John Wiley \& Sons. 75-81.

18. Software \& Engineering Associates (SEA) Inc. 2007. Two-dimensional kinetics (TDK) nozzle performance computer program user's manual. 4-19. 